\section{Neuer Tyrosinkinaseinhibitor bei RCC geprüft}

\begin{abstract}
Cabozantinib verbesserte in der Phase-III-Studie METEOR das progressionsfreie Überleben von Patienten mit fortgeschrittenem Nierenzellkarzinom. Nun gibt es Resultate zum Gesamtüberleben.
\end{abstract}

abozantinib ist ein Tyrosinkinaseinhibitor (TKI), der u. a. MET, VEGFR und AXL hemmt. In der METEORStudie wurden Wirksamkeit und Sicherheit von Cabozantinib im Vergleich zum mTOR-Inhibitor Everolimus bei Patienten mit fortgeschrittenem oder metastasiertem Nierenzellkarzinom (RCC) und Progression nach Vorbehandlung mit einem VEGFR-TKI untersucht. 658 Patienten erhielten randomisiert Cabozantinib (60 mg/Tag) oder Everolimus (10 mg/ Tag). Primärer Endpunkt war das progressionsfreie Überleben (PFS). Die Auswertung hierzu ergab ein signifikant längeres PFS unter Cabozantinib im Ver- gleich zu Everolimus (median 7,4 vs. 3,8 Monate; Hazard Ratio [HR] 0,58; $\mathrm{p}<0,001)$. Die finalen Ergebnisse zum Gesamtüberleben (OS) basieren auf einer nicht geplanten zweiten Zwischenauswertung. Nach einem Follow-up von median knapp 19 Monaten lag das mediane OS im Cabozantinib-Arm bei 21,4 und im Everolimus-Arm bei 16,5 Monaten (HR 0,66; $p=0,00026)$. Die Therapie mit Cabozantinib verbesserte zudem die objektive Ansprechrate (17 vs. 3\%; $\mathrm{p}<0,0001)$. Dabei profitierten alle untersuchten Subgruppen gleichermaßen.

Das Sicherheitsprofil entsprach den bisherigen Beobachtungen. Die häufigs- ten Grad-3/4-Toxizitäten waren Hypertonie (15 vs. $4 \%$ ), Diarrhö (13 vs. $2 \%$ ), Fatigue (11 vs. $7 \%$ ), palmar-plantares Erythrodysästhesie-Syndrom (8 vs. $1 \%$ ), Anämie (6 vs. $17 \%$ ), Hyperglykämie (1 vs. $5 \%$ ) und Hypomagnesämie (5 vs. $0 \%$ ). Dabei war die Rate insgesamt in beiden Armen ähnlich (39 vs. $40 \%$ ). Unter Cabozantinib gab es 1 therapiebedingten Todesfall, unter Everolimus 2.

Fazit: Bei vorbehandelten Patienten mit fortgeschrittenem RCC verlängerte die Therapie mit Cabozantinib PFS sowie OS und verbesserte das objektive Ansprechen gegenüber Everolimus. Daher sollte Cabozantinib als neue Standardtherapie für diese Patienten betrachtet werden.

Choueiri TK et al. Cabozantinib versus everolimus in advanced renal cell carcinoma (METEOR): final results from a randomised, open-label, phase 3 trial. Lancet Oncol. 2016;17(7):917-27.
Judith Neumaier

\section{mCRPC: Radium-223 auch kombinierbar}

\section{Radium-223 verbesserte in der ALSYMPCA-Studie das Gesamtüberleben von Patienten mit kastrationsresistentem Prostatakarzinom und symptomatischen Knochenmetastasen deutlich im Vergleich zu Placebo. Nun liegen Daten zur Kombination von Radium-223 u. a. mit Abirateron oder Enzalutamid vor.}

$V^{\prime}$ or der Zulassung von Radium-223 (Ra-223) wurde eine internationale Phase-IIIb-Studie aufgelegt, um weitere Daten zu Sicherheit und Gesamtüberleben zu sammeln. Von 839 Patienten mit histologisch oder zytologisch bestätigtem kastrationsresistentem Prostatakar- zinom (CRPC) mit $\geq 2$ Knochenmetastasen (ohne viszerale Erkrankung, Lymphknotenmetastasen erlaubt) erhielten 696 Patienten mind. 1 und bis zu 6 Injektionen Ra-223; sie wurden in die Studie aufgenommen. Nach median 7,5 Monaten betrug das mediane Gesamt-

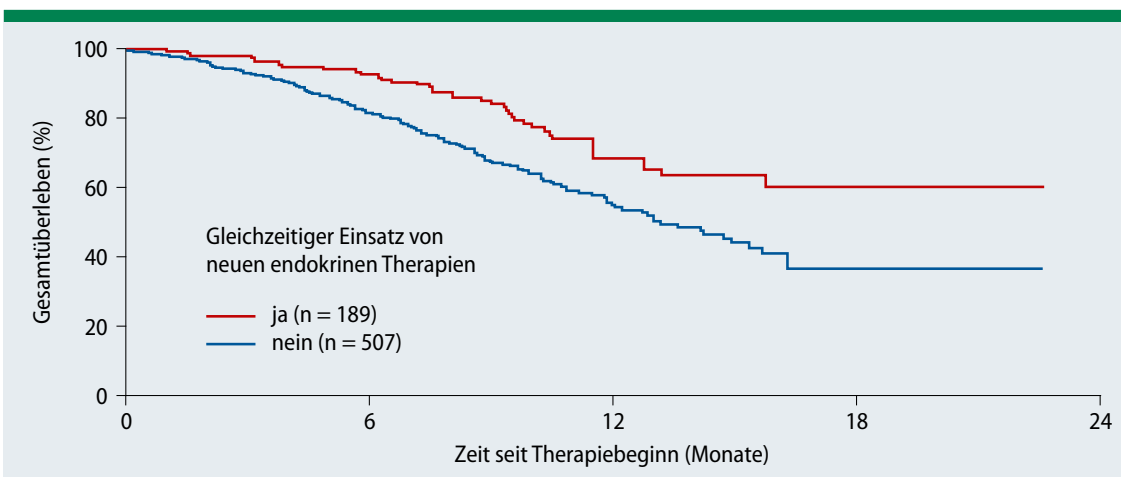

Abb. 1: Erhielten Patienten auch endokrine Therapien, verbesserte dies das Überleben. überleben (OS) 16 Monate, in Post-hocAnalysen war es u. a. länger bei Patienten mit folgenden Merkmalen zu Studienbeginn:

_alkalische Phosphatase (AP) im Normbereich versus höhere AP (Median nicht erreicht [n.e.] vs. 12 Monate), -ECOG-Performancestatus von 0 versus 1 oder $\geq 2$ (n.e. vs. 13 vs. 7 Monate) - keine Schmerzen versus leichte bzw. moderate bis schwere Schmerzen (n. e. vs. 14 vs. 11 Monate).

_Erhalt von Ra-223 plus Abirateron, Enzalutamid oder beides versus nur Ra-223 (n.e. vs. 13 Monate) (Abb. 1), _Erhalt von Ra-223 plus Denosumab versus nur Ra-223 (n.e. vs. 13 Monate). Therapiebedingte unerwünschte Ereignisse jedweden Grades traten bei $40 \%$ der Patienten auf.

Fazit: Ra-223 kann sicher mit z. B. endokrinen Therapien kombiniert werden. Die Daten zur Kombination sollten aber prospektiv randomisiert bestätigt werden. Judith Neumaier

Saad F et al. Radium-223 and concomitant therapies in patients with metastatic castration-resistant prostate cancer: an international, early access, open-label, single-arm phase $3 \mathrm{~b}$ trial. Lancet Oncol. 2016;17(9):1306-16. 